Monatsschr Kinderheilkd 2011 · 159:317-318 DOI 10.1007/s00112-010-2317-y

Online publiziert: 31. März 2011

(c) Springer-Verlag 2011

\author{
G. Staatz ${ }^{1}$ F. Zepp ${ }^{2}$ \\ ${ }^{1}$ Sektion Kinderradiologie, Klinik und Poliklinik für Diagnostische \\ und Interventionelle Radiologie, Universitätsmedizin Mainz \\ ${ }^{2}$ Zentrum für Kinder- und Jugendmedizin, Universitätsmedizin Mainz
}

\title{
Moderne Bildgebung in der Kinder- und Jugendmedizin
}

Der technische Fortschritt der radiologischen bildgebenden Verfahren hat die Möglichkeiten und die Qualität der diagnostischen Bildgebung in der Kinderradiologie in den vergangenen Jahrzehnten eindrucksvoll gewandelt. Neben dem konventionellen Röntgen, der Durchleuchtung und der Sonographie wurden in den letzten Jahren zunehmend moderne Schnittbildverfahren wie die MDCT und die MRT zur Diagnostik der spezifischen Erkrankungen im Kindes- und Jugendalter eingesetzt.

Die Anwendung moderner Bildgebungsmodalitäten im Kindesalter erfordert neben dem speziellen radiologischen Fachwissen, das im Anschluss an die Ausbildung zum Facharzt für diagnostische Radiologie in einer dreijährigen Weiterbildungszeit zum Facharzt für Kinderradiologie gewonnen werden kann, profunde Kenntnisse in der pädiatrischen Krankheitslehre. Zurzeit gibt es in Deutschland weniger als 80 hauptamtlich tätige Kinderradiologen, und vielerorts fehlt der interessierte Nachwuchs, um freie Stellen zu besetzen. Ungeachtet dessen entwickelt sich die Kinderradiologie kontinuierlich weiter, wie die Beiträge im vorliegenden Heft der Monatsschrift Kinderheilkunde demonstrieren. Auf dem Boden klinisch orientierter kinderradiologischer Forschung konnten interessante und vielversprechende moderne Bildgebungstechniken in verschiedenen pädiatrischen Fachgebieten für die Anwendung bei Kindern und Jugendlichen erschlossen werden.
Der Beitrag von PD Dr. Rainer Wunsch aus der Vestischen Kinder- und Jugendklinik in Datteln gibt einen Überblick über neue Ultraschalltechniken, die eine deutliche Artefaktreduktion bei gleichzeitiger Verbesserung der Darstellbarkeit von anatomischen Strukturen ermöglichen. „Tissue harmonic imaging" ermöglicht durch selektive Nutzung von Oberwellen im Echosignal der Ultraschallwellen eine Verbesserung des Bildkontrasts, welches insbesondere für den MagenDarm-Ultraschall hilfreich ist. Die Crossbeam-Technik erlaubt durch eine multidirektionale Abtasttechnik, gekrümmt oder geschlängelt verlaufende Strukturen besser abzubilden, was z. B. bei der Beurteilung der Gallenblasenwand nützlich ist. "Speckle reduction imaging" (SRI) verringert die artifizielle Körnigkeit in der Gewebedarstellung, wodurch kleinste Inhomogenitäten des Parenchyms besser abgebildet werden können, und z. B. Parenchymläsionen deutlicher sichtbar sind.

Die Arbeitsgruppe um Prof. Dr. H.J. Mentzel aus dem Universitätsklinikum Jena stellt ihre langjährigen Erfahrungen mit der diffusionsgewichteten MRT des Gehirns bei Kindern vor. Mit dieser innovativen Technik lassen sich nicht nur hypoxisch-ischämische Hirnerkrankungen frühzeitig gut erfassen, sondern es können zusätzlich wichtige Informationen bei Tumorerkrankungen und Entzündungen des Gehirns gewonnen werden.

"Diffusion tensor imaging" (DTI) erlaubt es, die Beschaffenheit bzw. die Integrität der weißen Substanz und den Ver- lauf der Faserorientierung von Nervenfaserbündeln darzustellen und zu beurteilen, was auch die Grundlage für die Traktographie („fiber tracking“) bildet. Die Traktographie kann zur Operationsplanung und Verlaufsbeurteilung von Gehirntumoren, zur Beurteilung der Hirnentwicklung bei verschiedenen Stoffwechselerkrankungen und Leukodystrophien im Kindesalter sowie zur Diagnostik von Hirnfehlbildungen genutzt werden. In $\mathrm{Zu}$ kunft dürfte die Traktographie aber auch bei Kindern mit entwicklungspsychologischen und kinderpsychiatrischen Fragestellungen, wie z. B. bei der Lese-Rechtschreib-Schwäche, dem Autismus oder dem Aufmerksamkeitsdefizit-Hyperaktivitäts-Syndrom, Anwendung finden.

Die klinischen Anwendungsgebiete der kardiovaskulären MRT im Kindesalter werden von der Arbeitsgruppe um Dr. H. Neubauer und Prof. Dr. M. Beer vorgestellt. Anhand von Fallbeispielen werden wichtige Indikationen zur pädiatrischen KardioMRT, wie die morphologische und funktionelle Abklärung kongenitaler Herzfehler, der Myokarditis und der Ischämie sowie von Erkrankungen der Koronararterien, erläutert. Die Vorteile des Verfahrens ergeben sich aus der umfassenden Beurteilung von Morphologie, Funktion und Metabolismus des Herzens ohne Strahlenbelastung. Limitationen bestehen für die Darstellung kleiner intrathorakaler Gefäßstrukturen wie z. B. aortopulmonaler Kollateralen (MAPCA), und für die Abbildung der Pulmonalvenen, die weiterhin eine Domäne der Computertomographie sind. 
Die Computertomographie hat sich in den letzten Jahren technisch enorm weiterentwickelt und ist in der Kinderradiologie trotz Strahlenbelastung immer noch für bestimmte Fragestellung unverzichtbar. PD Dr. C. Heyer aus dem Berufsgenossenschaftlichen Universitätsklinikum Bergmannsheil der Ruhr-Universität Bochum gibt einen Überblick über die strengen Indikationsstellungen für die MDCT im Kindesalter. Diese umfassen im Wesentlichen die oben angesprochenen kardialen Fragestellungen, die hochauflösende Lungenbildgebung, die Akutdiagnostik von (poly-)traumatisierten Patienten und CT-gesteuerte Interventionen. Besonderes Augenmerk ist auf die Dosisreduktion durch Verwendung pädiatrisch orientierter Untersuchungsprotokolle zu lenken. Mittels solcher Niedrigdosisprotokolle lassen sich die Strahlenbelastung und das Risiko für ein strahleninduziertes Malignom bei Kindern deutlich reduzieren.

Die Arbeitsgruppe von Dr. S. Koeppe und PD Dr. J. Schäfer aus der Universitätsklinik Tübingen stellt ihre umfassenden Erfahrungen zur Lungen-MRT bei Kindern vor. Durch die heutzutage deutlich verbesserte MR-Technologie lassen sich nicht nur morphologische Aussagen bei infiltrativen und chronischen Lungenerkrankungen im Kindesalter treffen, sondern auch funktionelle Aspekte der Lungenperfusion und Lungenventilation beleuchten. Wichtiges Einsatzgebiet der Lungen-MRT ist auch die pränatale Erfassung angeborener pulmonaler Fehlbildungen, welche in den großen Zentren mittlerweile zum Standard der fetalen Diagnostik gehört. Die Ventilations- und Perfusions-MRT der Lunge ist für pädiatrische Patienten noch im Forschungsstadium; hier sind aber für die nächsten Jahre interessante neue Entwicklungen zu erwarten.

In den vergangenen 30 Jahren hat sich die Kinderradiologie zu einer technologisch ausgereiften, innovativen Disziplin entwickelt und ist heute für die Kinderund Jugendmedizin mit Ihren vielfältigen Spezialdisziplinen ein unverzichtbarer Partner in Diagnostik und Therapiebegleitung geworden. Die Möglichkeit, mit modernen bildgebenden Verfahren nicht nur anatomische Strukturen darzustellen, sondern zeitnahe Funktionsana- lysen durchführen zu können, stellt für die moderne Kinder- und Jugendmedizin einen technologischen Quantensprung dar. Gerade bei der Beurteilung von Entwicklungs- und Differenzierungsstörungen im frühen Kindesalter kommt den belastungsarmen Technologien wie Ultraschall und MRT eine herausragende Bedeutung zu. Keine andere Patientengruppe profitiert so eindrücklich vom Fortschritt moderner Bildgebung, und für keine andere Patientengruppe ist der Einsatz strahlenarmer bzw. strahlenfreier Bildgebungsverfahren von so essenzieller Bedeutung. Moderne Kinderradiologie ist nicht nur Teil des medizinischen Fortschritts in unserem Fach sondern auch Garant für die Weiterentwicklung einer klinisch-wissenschaftlichen Kinder- und Jugendmedizin.

Wir hoffen, dass es uns mit diesem Leitthemenheft gelungen ist, die Vielfalt und die Bedeutung moderner kinderradiologischer Bildgebung für die Kinderund Jugendmedizin darzustellen und viele junge Kolleginnen und Kollegen für diese spannende Partnerdisziplin zu begeistern.

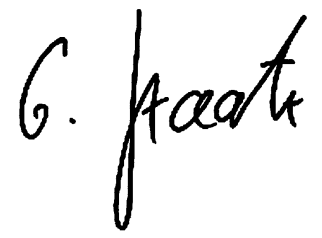

Prof. Dr. Gundula Staatz

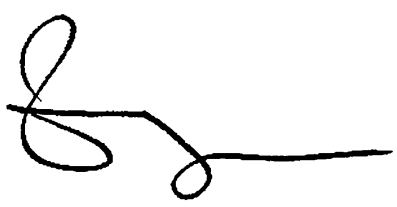

Prof. Dr. Fred Zepp

\section{Korrespondenzadresse}

Prof. Dr. G. Staatz

Sektion Kinderradiologie, Klinik und Poliklinik für Diagnostische und Interventionelle Radiologie, Universitätsmedizin Mainz Langenbeckstr. 1, 55131 Mainz staatz@radiologie.klinik.uni-mainz.de
Transitionsnephrologie Übergang von der pädiatrischen in die internistische Nephrologie

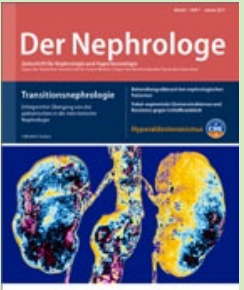

Eine gute interdisziplinäre Kooperation ist vor allem in der Kinderheilkunde und Jugendmedizin sehr wichtig wenn das chronisch kranke Kind aus seinen

Kinderschuhen herausgewachsen ist und die Weiterbetreuung durch Nachbardisziplinen, in aller Regel der internistischen, zu erfolgen hat. Gerade in der Nephrologie kommt dieser Übergangsphase eine besondere Bedeutung zu.

In der Ausgabe 01/2011 der Zeitschrift „Der Nephrologe sind Themen herausgearbeitet, die sowohl der Vermittlung der medizinischen und psychosozialen Hintergründe gelten als auch den Überlegungen zu deren praktischer Durchführung. Ziel ist es, die Übergangsphase und die Kooperation zwischen Pädiatrie und Innerer Medizin noch besser zu gestalten.

Das Heft mit dem Leitthema „Transitionsnephrologie" beinhaltet u.a. Beiträge zu folgenden Themen:

- Pädiatrisch nephrologische Erkrankungen nach dem Transfer

- Somatische Reifung des nierenkranken Kindes

- Chronische Nierenerkrankung in der Adoleszenz - Psychologische Aspekte

- Strukturelle Probleme der Transition

- Mögliche Ansätze für eine gute Transition

Bestellen Sie diese Ausgabe zum Preis von EUR 34,- zzgl. Versandkosten bei:

Springer Customer Service Center GmbH Kundenservice Zeitschriften

Haberstr. 7

69126 Heidelberg

Tel.: +49 6221-345-4303

Fax: +49 6221-345-4229

leserservice@springer.com

www.DerNephrologe.de 